\title{
The eclipsing, double-lined, Of supergiant binary Cygnus OB2-B17
}

\author{
V. E. Stroud ${ }^{1,2,3}$, J. S. Clark ${ }^{2}$, I. Negueruela ${ }^{4}$, P. Roche ${ }^{1,2,3}$, A. J. Norton ${ }^{2}$, and F. Vilardell ${ }^{4}$ \\ 1 Faulkes Telescope Project, School of Physics and Astronomy, Cardiff University, Cardiff, CF24 3AA, UK \\ e-mail: vanessa.stroud@faulkes-telescope.com \\ 2 Department of Physics and Astronomy, The Open University, Walton Hall, Milton Keynes MK7 6AA, UK \\ 3 Division of Earth, Space and Environment, University of Glamorgan, Pontypridd, CF37 1DL, UK \\ 4 Departamento de Física, Ingeniería de Sistemas y Teoría de la Señal, Universidad de Alicante, Apdo. 99, 03080 Alicante, Spain
}

Received 20 March 2009 / Accepted 15 December 2009

\section{ABSTRACT}

\begin{abstract}
Context. Massive, eclipsing, double-lined, spectroscopic binaries are not common but are necessary to understand the evolution of massive stars as they are the only direct way to determine stellar masses. They are also the progenitors of energetic phenomena such as X-ray binaries and $\gamma$-ray bursts.

Aims. We present a photometric and spectroscopic analysis of the candidate binary system Cyg OB2-B17 to show that it is indeed a massive evolved binary.

Methods. We utilise $V$ band and white-light photometry to obtain a light curve and period of the system, and spectra at different resolutions to calculate preliminary orbital parameters and spectral classes for the components.

Results. Our results suggest that B17 is an eclipsing, double-lined, spectroscopic binary with a period of $4.0217 \pm 0.0004$ days, with two massive evolved components with preliminary classifications of $\mathrm{O} 7$ and $\mathrm{O} 9$ supergiants. The radial velocity and light curves are consistent with a massive binary containing components with similar luminosities, and in turn with the preliminary spectral types and age of the association.
\end{abstract}

Key words. binaries: eclipsing - binaries: spectroscopic - stars: early-type - stars: fundamental parameters - stars: evolution stars: individual: Cyg OB2 B17

\section{Introduction}

O stars are amongst the most massive and intrinsically luminous stellar objects found in galaxies. Since they are the only direct way to measure the masses and radii of stars, binaries are the perfect testbeds for studying the physical properties and evolution of such stars. Unfortunately, as reported by Bonanos (2009), less than $20 \mathrm{O}$ stars have accurate $(\leq 10 \%)$ dynamical mass estimates. Because of the scarcity of known massive, eclipsing, doublelined, spectroscopic binaries - and hence dynamical mass estimates for stars at different evolutionary states (e.g., Gies 2002) the mass luminosity relation and theoretical evolutionary tracks of massive stars $\left(M \geq 20 M_{\odot}\right)$ are currently poorly constrained by observations.

In order to address this shortfall, much effort has been expended to identify further examples, utilising photometric and spectroscopic observations of young massive clusters such as the Arches (Martins et al. 2008), Quintuplet (Figer et al. 1999), and Westerlund 1 (Clark et al. 2005; Ritchie et al. 2009). These observations indicate that the binary fraction is potentially very high ${ }^{1}$; Kobulnicky \& Fryer (2007) inferred it to be $\geq 80 \%$ in Cygnus OB2 (Cyg OB2), while Clark et al. (2008) estimate the binary fraction of WR stars in Westerlund 1 to be $\geq 70 \%$. Currently the stars with the highest dynamical mass estimates are the twin WN6ha components of WR20a within Westerlund $2\left(83 M_{\odot}+82 M_{\odot}\right.$, Rauw et al. 2004; Bonanos et al. 2004) and the newly discovered WN6ha binary NGC3603-A1

\footnotetext{
1 A large amount of observations and patience are necessary to determine the true binarity of a sample of stars in an open cluster as observed in Sana et al. (2008).
}

$\left(116 \pm 31 M_{\odot}+89 \pm 16 M_{\odot} ;\right.$ Schnurr et al. 2008). This in turn has implications for the determination of the initial mass function (IMF) for the clusters in question and, by extension, the empirically determined maximum mass possible for a star (cf. Figer 2005). Moreover, massive close binaries are the progenitors of such diverse energetic phenomena as supernovae, $\gamma$-ray bursts and X-ray binaries (Ribas 2006). Clearly the properties of the progenitor binary population must be known to constrain their formation channels.

Cyg OB2 is one of the most massive and richest associations in the Galaxy. It is $\sim 2 \mathrm{Myr}$ old and $1.8 \mathrm{kpc}$ away (Kiminki et al. 2007). Containing at least 60-70 O-type stars (Negueruela et al. 2008), its proximity and accessibility to optical studies have made it the focus of numerous observational campaigns to determine the properties of its massive stellar population (e.g. Massey \& Thomson 1991; Knödlseder 2000; Hanson 2003; Comerón et al. 2002; Kiminki et al. 2007, 2008).

Cyg OB2 B17 (Comerón et al. 2002; henceforth B17 and also known as V1827Cyg, 2MASS J20302730+4113253, NSVS 5738756; $\alpha_{2000}=20^{\mathrm{h}} 30^{\mathrm{m}} 27.3^{\mathrm{s}}, \delta_{2000}=+41^{\circ} 13^{\prime} 25^{\prime \prime}$; $V=12.6$ ) is a luminous, variable member of the Cyg OB2 association. Comerón et al. (2002) observed the system as part of a near-infrared spectroscopic survey and found the spectrum presented $\mathrm{Br} \gamma$ emission, confirming it to be an evolved massive star. Follow up observations were made by Negueruela et al. (2008), who found it to stand out from the rest of the members due to its variability and strong emission lines. They classified it as an Ofpe star and suggested it was a strong binary candidate.

This paper reports the first results of an extensive multiepoch photometric and spectroscopic observational campaign on 
the binary candidate B17. Section 2 gives a description of the photometric and spectroscopic observations. Photometrically, the system was found to be variable and we report the analysis of the light curve in Sect. 3. More spectroscopic data were obtained permitting preliminary spectral and luminosity classification of the system; this analysis is shown in Sect. 4, along with descriptions of the long and short timescale variations of the spectra. The light and radial velocity curve modelling is presented in Sect. 5. A discussion of the system, including its evolutionary status, is found in Sect. 6 and a summary is presented in Sect. 7.

Note that the central goals of this manuscript are to verify the binary hypothesis and present a preliminary spectral classification. A full analysis of the system, consisting of the deconvolution of an expanded spectral data set and subsequent model atmosphere analysis to determine the fundamental stellar parameters of the system will be presented in a future paper (Stroud et al., in prep).

\section{Observations and data reduction}

\subsection{Photometry}

The North Sky Variability Survey (NSVS, Wózniak et al. 2004) is a record of the sky at declinations higher than $\delta=-38^{\circ}$ over the optical magnitude range 8 to 15.5 . It contains light curves of over 14 million objects. The data were taken between 1999 April and 2000 March by the first-generation Robotic Optical Transient Search Experiment (ROTSE-I) at Los Alamos National Observatory, New Mexico. The telescope consisted of four unfiltered Canon $200 \mathrm{~mm}$ telephoto lenses with $f / 1.8$ focal ratio, each covering $8.2 \times 8.2$. These were equipped with AP10-cameras and Thomson TH7899M CCDs. The lenses had a typical point-spread function with a full width half maximum of $\sim 20^{\prime \prime}$. In a median field, the bright unsaturated stars had a point-to-point photometric scatter of $\sim 0.02 \mathrm{mag}$ and position errors within $2^{\prime \prime}$. The calibrated images were passed through SExtractor software (Bertin \& Arnouts 1996), reducing them to object lists. The data were accessed through the Sky Database for Objects in Time-Domain (SkyDOT) at Los Alamos National Laboratory. A total of 186 observations were obtained for B17.

Additional $V$ band photometry was obtained by amateur astronomers Pedro Pastor Seva (observer 1) and Manuel Mndez Marmolejo (observer 2) between 2007 April 03 and 2007 June 11. Observatory 1 is located in Muchamiel (Alicante, Spain). The telescope used was an 8 inch Vixen VISAC Schmidt-Cassegrain telescope. It has a field of view of $24^{\prime} \times 20^{\prime}$ and a focal ratio of $f / 8$. The telescope was equipped with a SBIG ST10-XME CCD chip. Observatory 2 is located in Rota (Cádiz, Spain). The telescope used was an 8 inch Meade LX200 Schmidt-Cassegrain telescope. It has a field of view of $16^{\prime} \times 12^{\prime}$ (as a smaller SBIG ST7-XME chip was used) and a focal ratio of $f / 6.3$. Exposures varied between 3 and 4 min giving a $S N R \sim 200$. The data were calibrated and analysed using Mira $\mathrm{Pro}^{2}$ and AIP4WINv $2^{3}$ packages. The apparent magnitudes were obtained using differential photometry with respect to a set of reference stars in the image with known magnitudes. In both cases the precision for the individual measure is always better than $0.01 \mathrm{mag}$.

\footnotetext{
${ }^{2}$ Mira Pro software is published by Mirametrics Inc., which has no connection with the Monterey Institute for Research Astronomy.

3 AIP4WINv2 is published by Willmann-Bell, Inc.
}

Table 1. Log of spectroscopic observations.

\begin{tabular}{ccccc}
\hline \hline Date & $\begin{array}{c}\text { Telescope } \\
\text { /Instrument }\end{array}$ & $\begin{array}{c}\text { Nominal } \\
\text { Dispersion } \\
(\AA / \text { pix })\end{array}$ & $\begin{array}{c}\text { Wavelength } \\
\text { Range }(\AA)\end{array}$ & Phase \\
\hline $04 / 07 / 18$ & Cassini/BFOSC & 6 & $3800-6400$ & 0.437 \\
$04 / 07 / 18$ & Cassini/BFOSC & 6 & $6100-8200$ & 0.450 \\
$06 / 06 / 11$ & WHT/ISISB & 0.86 & $3200-5200$ & 0.036 \\
$06 / 06 / 11$ & WHT/ISISR & 0.93 & $5400-8000$ & 0.036 \\
$06 / 08 / 18$ & WHT/ISISB & 0.86 & $3200-5200$ & 0.929 \\
$06 / 08 / 18$ & WHT/ISISR & 0.93 & $5400-8100$ & 0.931 \\
$06 / 09 / 10$ & INT/IDS & 0.9 & $3900-5000$ & 0.600 \\
$06 / 09 / 11$ & INT/IDS & 0.9 & $3900-5000$ & 0.840 \\
$06 / 09 / 11$ & INT/IDS & 0.9 & $3900-5000$ & 0.891 \\
$07 / 07 / 21$ & WHT/ISISB & 0.86 & $3600-5300$ & 0.738 \\
$07 / 07 / 21$ & WHT/ISISR & 0.93 & $5400-8300$ & 0.738 \\
$07 / 07 / 21$ & WHT/ISISR & 0.93 & $5400-8300$ & 0.740 \\
$07 / 07 / 21$ & WHT/ISISR & 0.93 & $5400-8300$ & 0.741 \\
$07 / 08 / 21$ & WHT/ISISB & 0.23 & $3900-4750$ & 0.380 \\
$07 / 08 / 21$ & WHT/ISISB & 0.23 & $3900-4750$ & 0.385 \\
$07 / 08 / 21$ & WHT/ISISB & 0.23 & $3900-4750$ & 0.397 \\
$07 / 08 / 21$ & WHT/ISISB & 0.23 & $3900-4750$ & 0.416 \\
$07 / 08 / 21$ & WHT/ISISB & 0.23 & $3900-4750$ & 0.436 \\
$07 / 08 / 21$ & WHT/ISISB & 0.23 & $3900-4750$ & 0.453 \\
$07 / 08 / 22$ & WHT/ISISB & 0.23 & $3900-4750$ & 0.629 \\
$07 / 08 / 22$ & WHT/ISISB & 0.23 & $3900-4750$ & 0.634 \\
$07 / 08 / 22$ & WHT/ISISB & 0.23 & $3900-4750$ & 0.648 \\
$07 / 08 / 22$ & WHT/ISISB & 0.23 & $3900-4750$ & 0.664 \\
$07 / 08 / 22$ & WHT/ISISB & 0.23 & $3900-4750$ & 0.683 \\
$07 / 08 / 22$ & WHT/ISISB & 0.23 & $3900-4750$ & 0.701 \\
\hline & & & & \\
\hline
\end{tabular}

\subsection{Spectroscopy}

The spectra were obtained from several telescopes during the course of $\sim$ three years. Table 1 lists the full set of observations. The first set was observed with the 1.52-m G. D. Cassini telescope at the Loiano Observatory (Italy) during the night of 2004 July 18. The telescope was equipped with the Bologna Faint Object Spectrograph and Camera (BFOSC) and an EEV camera. Grism 3 was used, which covers 3300-5800 $\AA$ with a resolution of $\sim 6 \AA$. More spectra of the system were obtained with the 4.2-m William Herschel Telescope (WHT), in La Palma (Spain) equipped with the ISIS double-beam spectrograph, during service runs on 2006 June 11 and August 18. The instrument was fitted with the R300R grating and MARCONI2 CCD in the red arm and the R300B grating and EEV12 CCD in the blue arm. Both configurations result in a nominal dispersion of $0.85 \AA /$ pixel (the resolution element is approximately 3 pixels in the blue and 2 pixels in the red).

The system was also observed with the $2.5 \mathrm{~m}$ Isaac Newton Telescope (INT) in La Palma on 2006 September 10-11. The telescope was equipped with the Intermediate Dispersion Spectrograph (IDS), fitted with a R632V grating and EEV10 CCD. Another 12 spectra were obtained during a dedicated run on 2007 August 21-22 at the WHT. The system was observed in the blue arm with grating R1200B (nominal dispersion of $\sim 0.23 \AA /$ pixel). All the spectra were reduced with the Starlink packages CCDPACK (Draper et al. 2000) and FIGARO (Shortridge et al. 1997) and analysed using FIGARO and DIPSO (Howarth et al. 1998).

\section{Photometry}

The Starlink software PERIOD (Dhillon et al. 2001) was used on both the NSVS and amateur photometry to search for a 

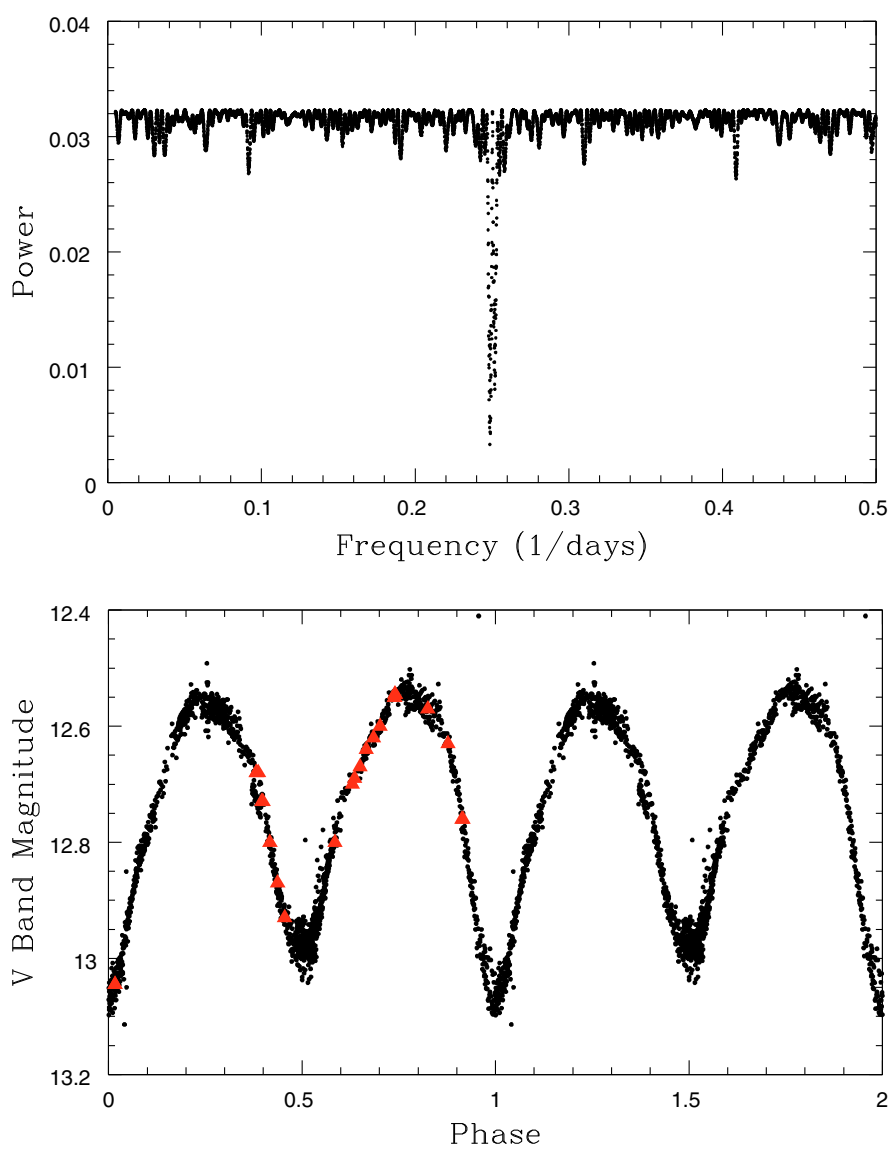

Fig. 1. Upper panel: periodogram for B17 data using the reduced- $\chi^{2}$ technique. Lower panel: light curve folded on a 4.0217 day period. The red triangles show the phases for which spectra were obtained.

modulation period in the photometric data, using phase dispersion minimisation, $\chi^{2}$ of sine fit vs. frequency and string-length vs. frequency methods. The resultant periods were consistent and favoured an orbital period of $4.0217 \pm 0.0004$ days (the error being estimated from the spread in the periods derived from the different methods employed). When both data sets were folded together they were found to be in phase; therefore, given that there appears to be no change of shape or shift in period in the 7 years between the NSVS and amateur observations, we are confident that the period determined is accurate to within the errors quoted.

Figure 1 shows the periodogram obtained using Period with the reduced- $\chi^{2}$ technique (top) and the $V$ band light curve folded on a 4.0217 day period, along with the phases of the spectra obtained (bottom).

The shape of the light curve suggests that the system is a semi-detached binary. Both minima are narrow and demonstrate different eclipse depths, although the 0.5-0.6 mag range indicates the almost complete eclipse of each star and hence that the two stars are of similar size but with different luminosities (in a contact system, the temperature of both stars should be the same). There appears to be an asymmetry on the light curve between phases $\phi=0.6-0.75$ which is observed in both sets of data. Hilditch et al. (2005) found similar depressions in eclipsing binaries in the Small Magellanic Cloud and Bonanos (2009) found a similar depression in the light curve for the binary LMCSC1-105. They attributed these asymmetries to the presence of a mass-transfer stream. Linder et al. (2009) also observed this asymmetry in the overcontact binary Cyg OB2 \#5 and attributed this to the secondary being brighter on its leading side due to the colliding wind region; first discussed by Rauw et al. (1999).

The quadratures display an O'Connell effect - where the maxima are of different brightnesses - of $\sim 0.02$ mag which is likely due to variations in the brightness of the stellar surface(s) due to mass transfer between the components.

The principal minimum was estimated using the amateur data to be at JD $=2454272.527 \pm 0.005$. From the light curve, the ephemeris for the primary eclipse was found to be:

$\min I=2454272.527+4.0217 E(\mathrm{JD})$

where $E$ is the number of orbital cycles after the given epoch.

We note that our period determination is in agreement with a value recently established by Otero (2008) using the same data from NSVS as used in this paper.

\section{Spectroscopy}

The spectrum of B17 is found to be highly variable on both short (<day) and long ( year) timescales, with the most prominent features in the spectra being $\mathrm{H}$, He I, He II and N III lines in absorption and He II and N III lines in emission. Wavelength shifts are observed for different lines, some of which become double in some spectra suggesting that it is a binary system with at least one hot component, as revealed by the presence of He II and $\mathrm{N}$ III.

\subsection{Long term variability}

Figure 2 shows blue-violet spectra (4000-4900 A) obtained at random phases between 2004 July and 2007 August. The most prominent elements observed are $\mathrm{H}$, He I, He II and N III in absorption and He II $\lambda 4686$ and N III $\lambda \lambda 4634-40-42$ in emission. The spectra show strong, highly variable N III and He II emission lines; in 2004 July 18 these are of a similar strength but by 2006 June 11 the He II line is almost three times the intensity of the N III lines. The N III profile is also variable; it appears as two separate peaks in some of the spectra (2006 June 11, 2006 September 10-11, 2007 July 21) and it is almost completely blended in the spectra taken on 2004 July 18 and 2006 August 18 , although this in part could be due to the low resolution of the spectrum from 2004 July 18.

The hydrogen lines are also highly variable; this is particularly evident for $\mathrm{H} \gamma$ when compared to the diffuse interstellar band (DIB) at $\lambda 4428 . \mathrm{H} \beta$ shows a P-Cygni profile varying in both width and strength suggesting the stellar wind is highly variable. Finally, the $\mathrm{He}$ I $\lambda 4471 / \mathrm{He}$ II $\lambda 4541$ ratio, which is employed as a standard diagnostic for the spectral type of early stars (Walborn \& Fitzpatrick 1990), is also observed to vary over the course of the observations.

The most prominent line in the yellow spectra (5600$6000 \AA$ ) is the varying P-Cygni profile in He I $\lambda 5875$, which blends with the Na I interstellar lines (Fig. 3). When compared to the nearby DIBs at 5780-5800 $\AA$, the absorption trough in He I line was observed to vary by a factor of 4 in intensity relative to the nearby DIB features, with the emission component also varying in strength by a factor of 2 . Other lines in the yellow spectra include C III $\lambda 5696$ and C IV around $\lambda 5810$.

The most prominent feature in the red spectra (6350$6650 \AA$ ) is the $\mathrm{H} \alpha$ line which also demonstrates a highly variable P-Cygni profile which is blended with He II absorption features in all the red spectra, complicating analysis of the profile. It has 


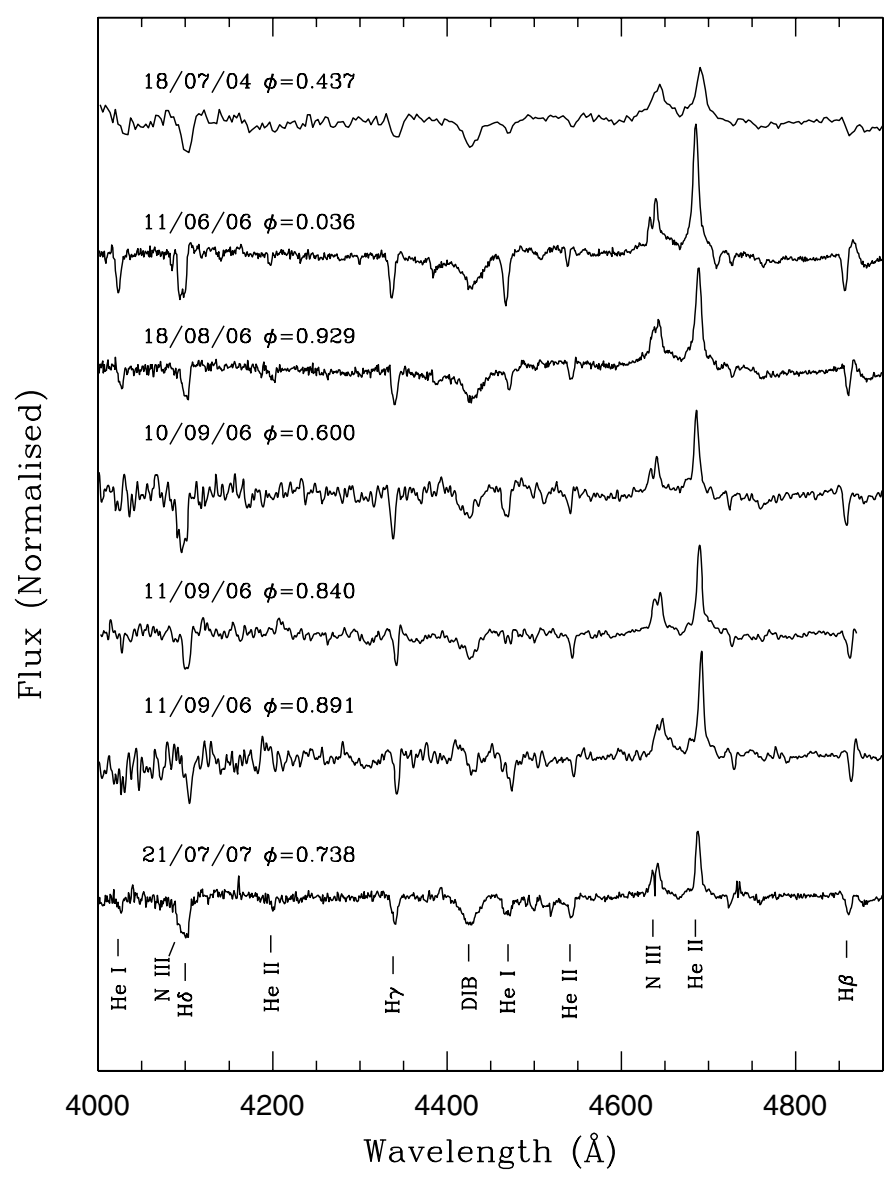

Fig. 2. Blue-violet spectra of B17 obtained at different phases over a three year period, with the most prominent lines labeled.

a double peak profile which varies in intensities with the redder peak being between 2 and 4 times stronger than the bluer peak. The depth of the He II line, which presumably contributes to the double peaked morphology is also variable (Fig. 4). Preliminary calculations for the velocities of the blue edge of the $\mathrm{H} \alpha$ profile $\left(v_{\text {edge }}\right)$ have a range of $2260-2625 \mathrm{~km} \mathrm{~s}^{-1}$ with an average $2440 \pm 50 \mathrm{~km} \mathrm{~s}^{-1}$. The $v_{\text {edge }}$ is related to the terminal velocity of the wind; in the extreme ultraviolet, the terminal velocities for OB stars are 15-20\% smaller than the edge velocities (Prinja et al. 1990). Variations in the wind profiles of OB stars are thought to be a consequence of highly structured winds on multiple scales, and while it appears likely that the variations observed for B17 arise due to a highly anisotropic circumstellar envelope observed at differing lines of sight throughout the orbital period, the current limited data set offers little prospect of a more explicit physical interpretation.

Unfortunately, no spectra with the same orbital phases have been obtained at different epochs and so it has not been possible to search for unambiguous long term secular variability in the current data set.

In Fig. 5 we plot the EW of the He II $\lambda 4686$ line against orbital phase, finding the EW to be broadly anticorrelated with the photometric light curve, increasing by a factor of two during the entry to eclipse. This result suggests that the strength of the line remained $\sim$ const. over the segments of the orbital period sampled by the observations, implying that the change in EW is primarily due to dilution by the variable continuum.

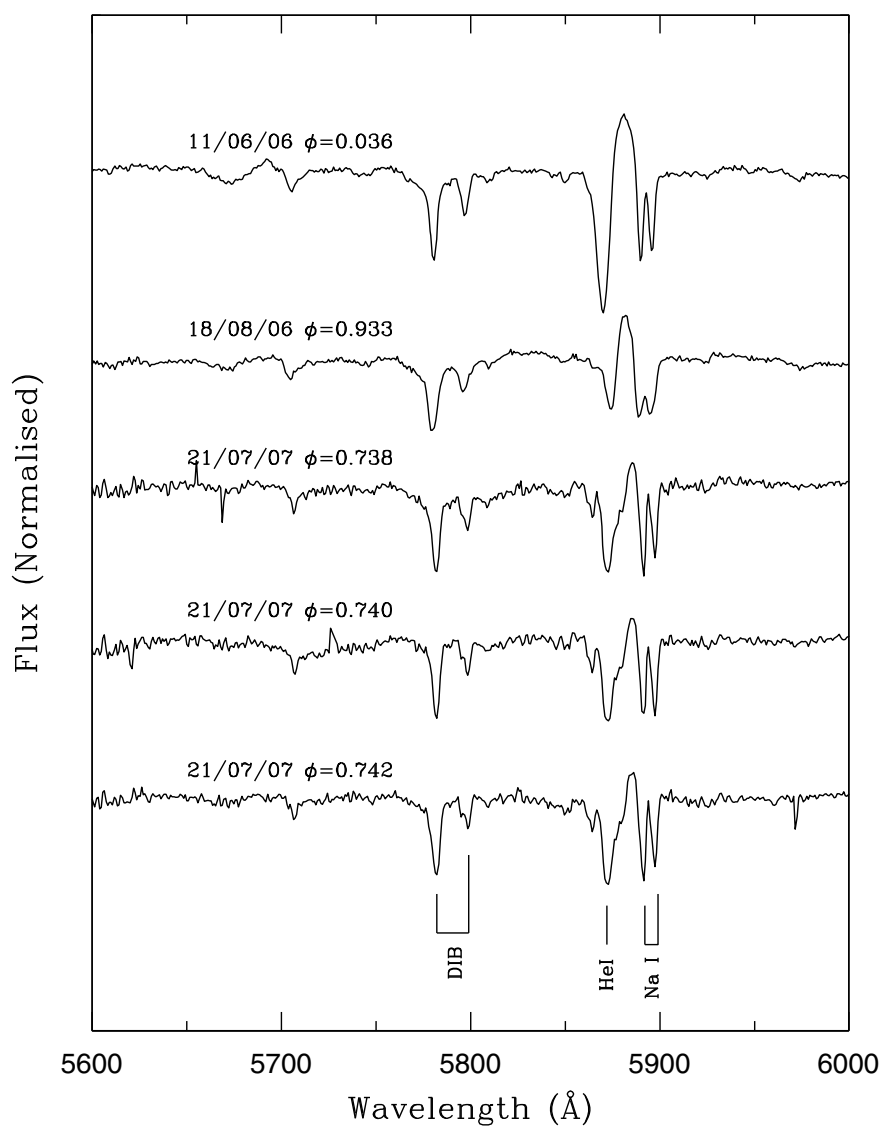

Fig. 3. B17 yellow spectra showing variability at different phases taken over a period of three years. Prominent lines are labeled.

\subsection{Short term variability}

The set of 12 high resolution spectra obtained during the nights of the 2007 August 21-22 cover the phases $\phi=0.38-0.453$ and $\phi=0.629-0.701$ of one period. The spectra obtained during the first night (Fig. 6 upper panel) have narrower $\mathrm{H}$ and He II absorption lines compared to the spectra from the second night. During the first night, the He I $\lambda 4471$ /He II $\lambda 4541$ ratio - used for classifying $\mathrm{O}$ stars - changes monotonically in the sense of increasing He I $\lambda 4471$ strength, reaching unity in the final spectrum.

A second, blueshifted absorption component appears on the shoulder of the He I $\lambda 4471$ line, becoming stronger with time; similar evolution is also observed in the $\mathrm{H} \delta$ profile. Initially, the N III $14634-40-42$ emission is highly blended, with the redshifted peak being the strongest. These peaks start to separate on the last spectrum of that night $(\phi=0.453)$. The He II $\lambda 4686$ emission feature appears to be non-symmetric and strengthens throughout the night. The He I $\lambda 4026, \mathrm{He}$ II $\lambda 4200$ and $\mathrm{H} \gamma$ lines do not vary significantly throughout the first night.

During the $17 \mathrm{hrs}$ between the last observation of night one $(\phi=0.453)$ and the first of night two $(\phi=0.629)$ the spectrum has clearly evolved. The He I $\lambda 4026$, He II $\lambda 4200$ and $\mathrm{H} \gamma$ lines are broader and non symmetrical. On the second night (Fig. 6 lower panel), the He I $\lambda 4471 / \mathrm{He}$ II $\lambda 4541$ ratio is $>1$ on the first spectrum of the second night and decreases until it reaches unity on the last spectrum $(\phi=0.701)$. Significant changes are apparent in the He I $\lambda 4471$ absorption profile, which is much broader and appears to be composed of two troughs, with the bluer trough being stronger. An additional, distinct weak blueshifted absorption line is also present and becomes 


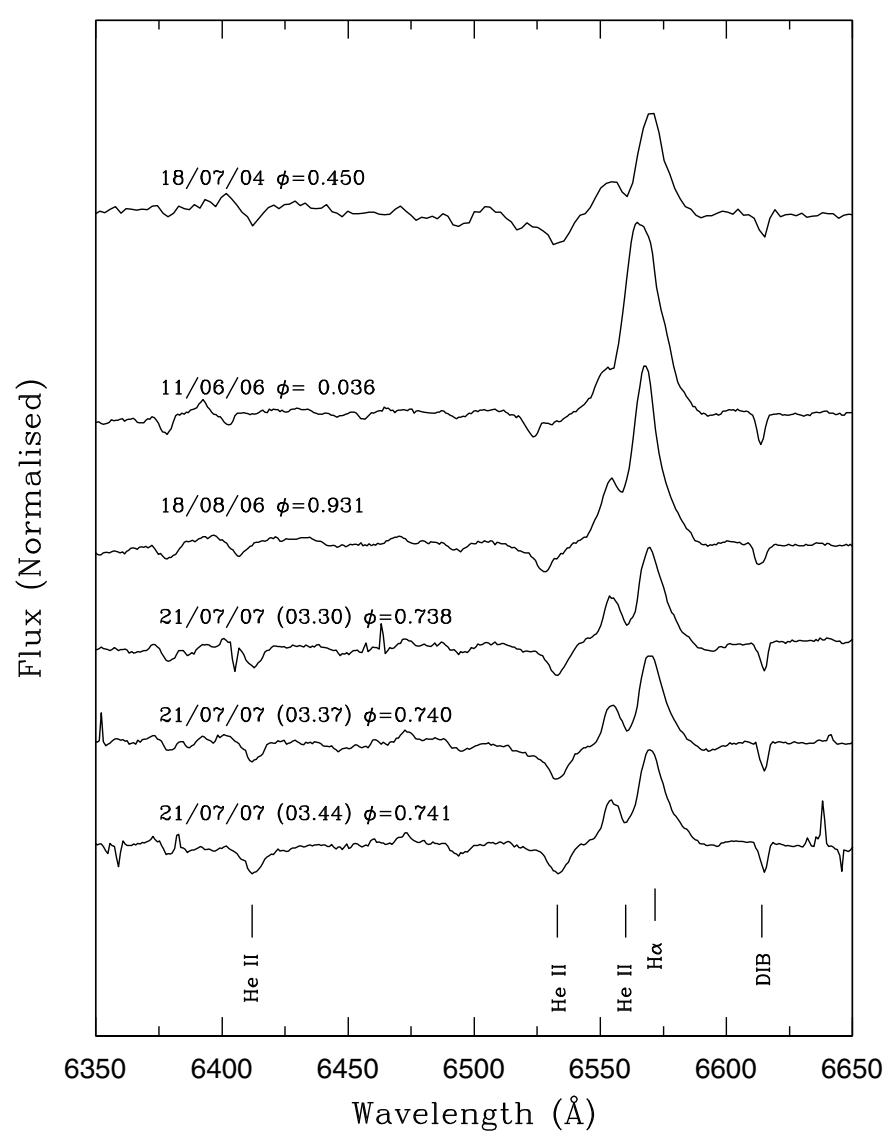

Fig. 4. B17 red spectra showing $\mathrm{H} \alpha$ variability at different phases taken over a period of three years. Prominent lines are labeled.

stronger with time. The $\mathrm{H} \delta$ line appears to be the result of the blending of two lines, showing a wide profile with two troughs. The bluer trough is stronger than the redder during the second night. The N III $\lambda 4634-40-42$ emission is observed as two separate peaks, with the redder being the strongest and the intensity of both increasing through the night. The He II $\lambda 4686$ emission feature appears to be symmetric compared to the first night and weakens throughout the night. We note that (as mentioned in Sect. 3) the light curve demonstrates an asymmetry - possibly due to mass transfer - between phases $\phi \sim 0.6-0.75$. Given this is coincident with the observations during night two, it is tempting to attribute the spectral changes (in part) to such a process.

\subsection{Spectral classification}

Given that the current spectroscopic dataset does not span an entire orbital cycle we have not attempted a formal deconvolution of the blended spectra. Instead we have simply used the spectra closest to the primary and secondary eclipses to perform a preliminary classification of the components of the system, using the stellar atlas of Walborn \& Fitzpatrick (1990) and Walborn \& Howarth (2000).

The spectrum at $\phi=0.453$ shows a He II $\lambda 4541 / \mathrm{He}$ I $\lambda 4471$ absorption-line ratio of one, suggesting a likely spectral classification of O7. Both He II $\lambda 4686$ and N III $\lambda \lambda 4634-40-42$ are in strong emission which is characteristic of an Of supergiant, implying an initial classification of $\mathrm{O} 7$ Iaf. Figure 7 upper panel shows this spectrum along with comparison spectra. The spectrum lacks the SiIV $\lambda 4089$ line, which is unexpected for an

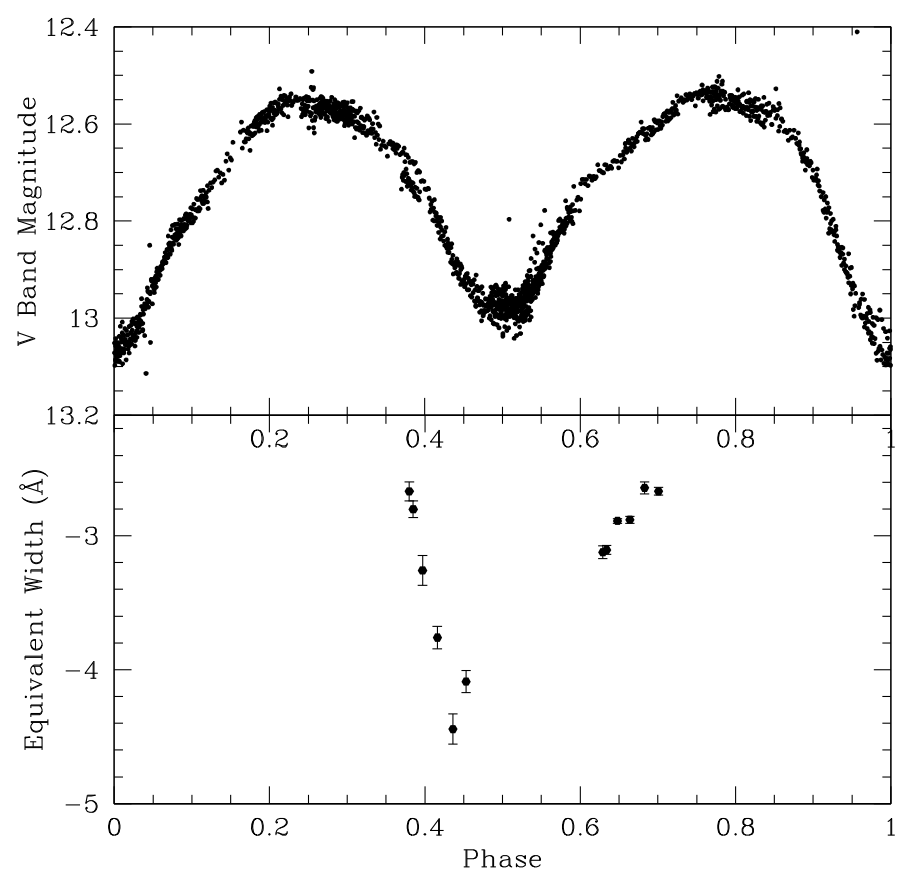

Fig. 5. Upper panel: light curve folded on a 4.0217 day period. Lower panel: equivalent widths of the He II $\lambda 4686$ emission line for the WHT data taken in 2007 August plotted on the same orbital period.

O7 SG ${ }^{4}$. Walborn (2001) mentions that a similar effect is found in spectra in the Small Magellanic Cloud where no Si IV $\lambda 4089$ is observed and he attributes this to the absorption and emission features cancelling each other. Therefore we suggest a preliminary spectral classification of $\mathrm{O} 7 \mathrm{Iaf}+$ for this component.

The spectrum at $\phi=0.036$ (Fig. 7, lower panel) shows a He II $\lambda 4541 / \mathrm{He}$ I $\lambda 4471$ absorption-line ratio smaller than one, indicating a spectral type later than O7. The He II $\lambda 4686$ and N III $\lambda \lambda 4634-40-42$ are also in strong emission, implying an Of supergiant. The spectrum also presents a stronger Si IV $4089 \mathrm{ab}-$ sorption line compared to the spectrum at $\phi=0.453$ although it is still weaker than expected for a late $\mathrm{O}$ star. Therefore we suggest a preliminary spectral classification of 09 Iaf for this component.

Since the combination of spectra of the preliminary classifications of an $\mathrm{O} 7$ and an $\mathrm{O} 9$ components would not give a HeI 4471/HeII 4541 ratio less than unity (see Sect. 4.2), it suggests there is emission in-filling of HeI 4471.

\subsection{Radial velocities}

The high resolution blue spectra show systematic night to night variations in the $\mathrm{H}, \mathrm{He}$ and $\mathrm{N}$ lines, revealing significant radial velocity shifts. Radial velocities were determined for the primary lines which appear not to be blended (He II $\lambda 4200$, He II $\lambda 4541$, $\mathrm{H} \gamma$ 14340) in the high resolution spectra obtained on the WHT observing run in 2007 August. Gaussians were fitted to the line profiles using the DIPSO emission line fitting command (ELF; the results are discussed in Sect. 5). The lines for the secondary appear to be blended and were not measured. The radial velocities for the secondary will be measured in the future paper after the spectra have been disentangled.

${ }^{4}$ We note that while this line is not present in the spectrum of the $\mathrm{O} 7$ supergiant Sanduleak 80, given the reduced metallicity appropriate for a SMC star it is not clear it provides a valid comparison. 

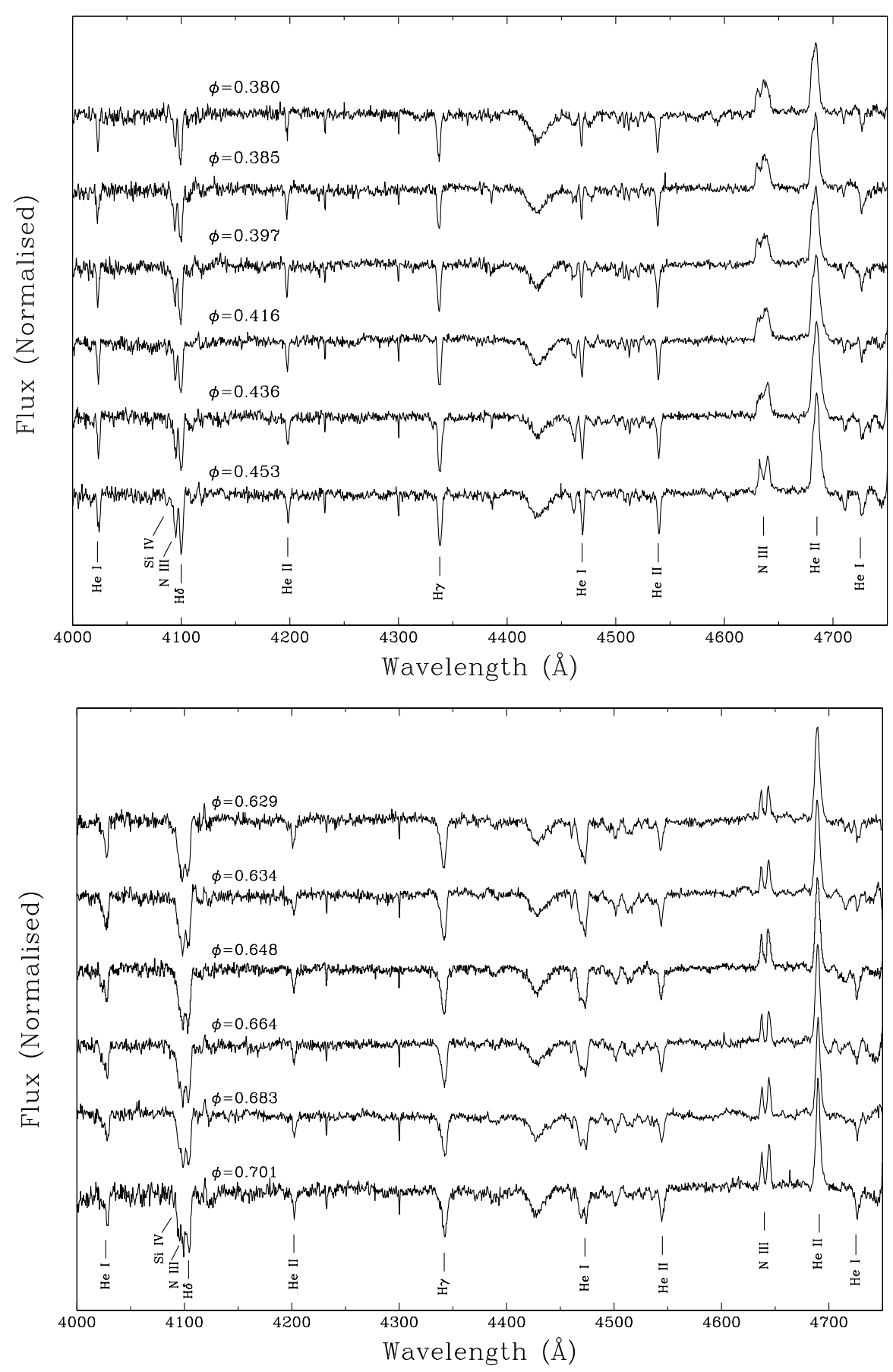

Fig. 6. Blue spectra obtained with the WHT on the night of 2007 August 21 and 22 . The main transition lines are shown. On the regions where the lines are blended, accuracy of the identified lines is not certain and will be clarified with spectra disentangling.

\section{Light and radial velocity curve analysis}

In order to reproduce the observed characteristics of the photometric light curve, we analysed it with the 2003 version of the Wilson \& Devinney (1971) W-D, code. For the analysis, the ROTSE (with an effective wavelength similar to Johnson $R$ ) and amateur $V$ light curves were modelled as different datasets. In all cases, detailed reflection-model and proximity-effect corrections were included. Considering the spectroscopic analysis, the temperature of the primary $\left(T_{\mathrm{eff}}^{\mathrm{P}}\right)$ was fixed to $35000 \mathrm{~K}$, and the bolometric albedo and gravity brightening coefficients were set to unity, as generally found for stars with radiative envelopes. In addition, a circular orbit was adopted, as suggested by the equal separation between both (primary and secondary) eclipses, and a rotation rate synchronized with the orbital period was assumed for both components. The fitting process was carried out iteratively until three consecutive solutions provided differential corrections for all the parameters smaller than twice their internal errors.

Considering the characteristics in the light curves described above, the first runs in the modelling with W-D assumed a semidetached configuration. Numerous attempts were performed with several mass ratios and with either the primary or the secondary component filling the Roche lobe. However, in all cases, the fits provided solutions where both stars tended to be 

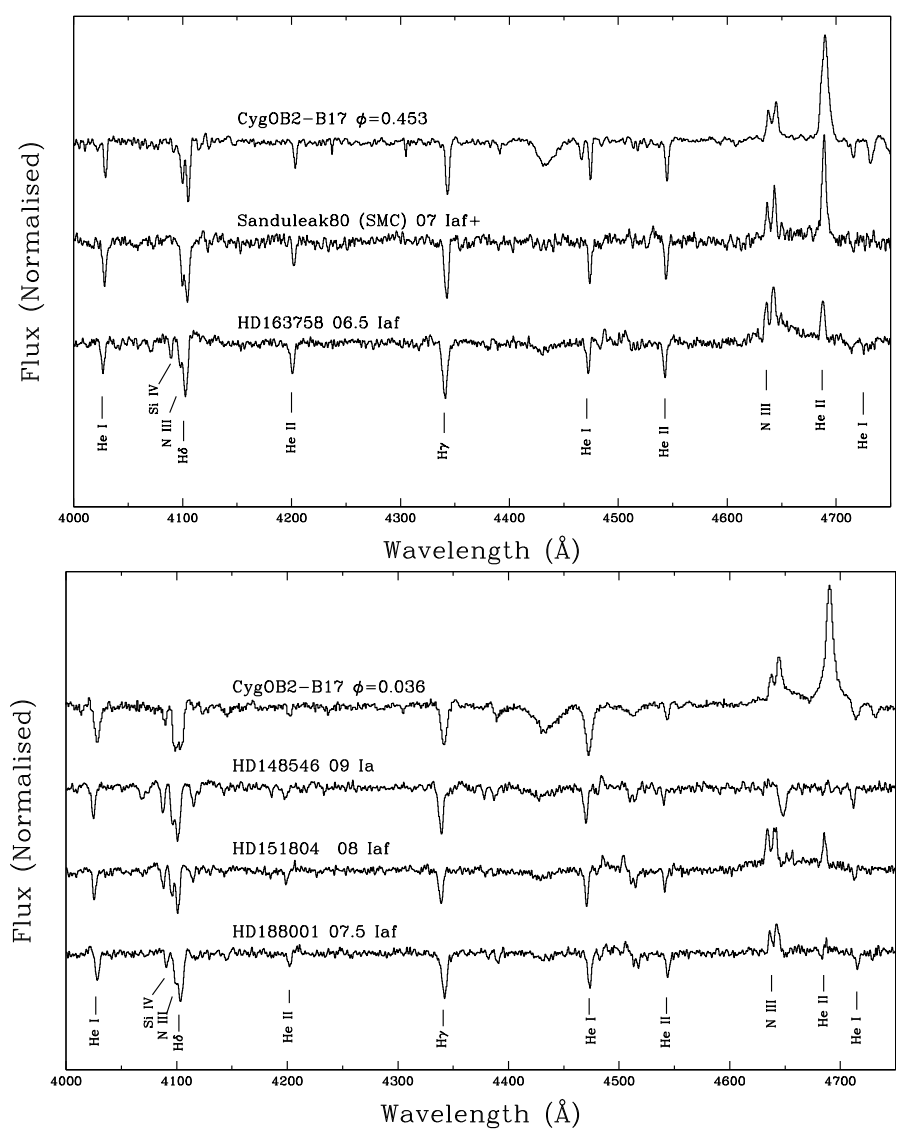

Fig. 7. Upper panel: B17 spectrum obtained with the WHT on the night of 2007 August 21, close to the secondary eclipse, compared with Of supergiant spectra from the Digital Atlas of Stellar Classification (Walborn \& Fitzpatrick 1990). The spectrum is most compatible with an O7Iaf classification. Double lines are observed even though the system is at eclipse which we currently cannot account for and will be further studied with the spectra disentangling. Lower panel: B17 spectrum obtained with the WHT on the night of 2006 June 11, close to the primary eclipse, compared with Of supergiant spectra from the Digital Atlas of Stellar Classification (Walborn \& Fitzpatrick 1990). The spectrum is more comparable to the $\mathrm{O} 9$ classification.

in contact. Therefore, despite the properties of the light curve suggesting a semi-contact configuration we finally attempted an over-contact solution - the results of which are described here where each component could have a different temperature, as observed from the different depths of the eclipses. For this model, the mass ratio was set to $q=0.75$ - as might be expected for $\mathrm{O} 7$ and $\mathrm{O} 9$ super-giant components - noting that the mass ratio cannot be smaller than $0.4^{5}$. The time of minimum $\left(t_{\min }\right)$, the period $(P)$, the orbital inclination $(i)$, the temperature of the secondary component $\left(T_{\text {eff }}^{\mathrm{S}}\right)$, the surface potential $\left(\Omega^{\mathrm{P}}\right)$ and the luminosity of the primary component $\left(L^{\mathrm{P}}\right)$ were all left as free parameters in the fit.

The $V$ light curve (which has smaller photometric errors) clearly reveals that one quadrature is $\sim 0.02$ mag brighter than the other. Two main solutions were attempted to explain the observed O'Connell effect, one with an equatorial hot spot $(30 \%$ hotter than the photosphere) on the primary component and another one with the spot being on the secondary component. The

\footnotetext{
${ }^{5}$ For a mass ratio of 0.4 , the implied mass of the primary -considering the minimum amplitude of the radial velocity curve- is over $120 M_{\odot}$, regardless of the light curve analysis.
}
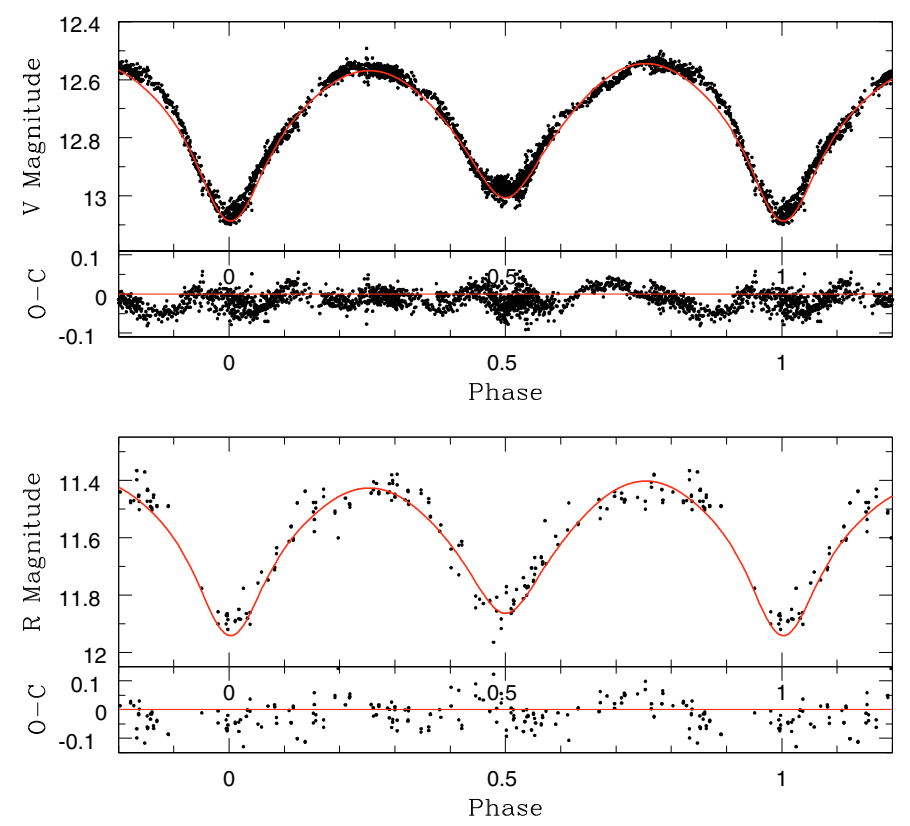

Fig. 8. The $V$ band and $R$ band light curves of B17 with the best fit model overlaid. The panels below the light curves show the deviations from the model.

size and position of the spot were left as free parameters and also fitted at each run, although we emphasise that the size and temperature of the spot are strongly correlated. The fits with the spot on the primary component were finally adopted, since they provided slightly smaller errors. In addition, the position of the spot (oriented roughly towards the secondary component) can more easily be explained as the interaction of the stellar winds.

Together with the light curve analysis, the radial velocities were also used to constrain the solution. Radial velocities were fitted separately from the light curve to avoid the larger number of photometric observations dominate in the solution; the parameters fitted being the semi-major axis $(a)$ and the systemic velocity $(\gamma)$. The rms of the fit is $9.5 \mathrm{~km} \mathrm{~s}^{-1}$ for the radial velocities, $0.023 \mathrm{mag}$ for the $V$ light curve and $0.048 \mathrm{mag}$ for the ROTSE light curve. Unfortunately, the accuracy of the solution obtained is strongly dependent on the mass ratio adopted. Nevertheless, Figs. 8 and 9 show the light curve and radial velocity curve with the best fit and Table 2 gives the parameters obtained from this solution.

Despite our efforts we are still not completely satisfied with this fit. In particular, we are still unable to adequately fit the egress from secondary minimum nor the subsequent light curve between phases $0.6-0.9$. Moreover it is expected that the temperatures of stars in over-contact binaries should be equal where a ratio of 0.85 was found between the secondary and primary. As such we regard the parameters presented in Table 2 as provisional at present. We anticipate that the determination of radial velocities for the secondary component, which will directly constrain the binary mass ratio will greatly clarify the fundamental properties of the components and the configuration of this EB system.

Finally, the light curve modelling, allows us to address the distance to B17, and by extension the Cyg OB2 association. Adopting the bolometric corrections from Martins et al. (2005) we may use the bolometric luminosities determined above (see Table 2) to calculate the absolute $V$ magnitudes of both components. The $V$ band reddening was then calculated by following a similar procedure used by Negueruela et al. (2008, see Sect. 6) 
Table 2. Results from the analysis of the light and radial velocity curves.

\begin{tabular}{lcc}
\hline \hline Parameter & Value \\
\hline$T_{0}$ (MJD) & $4272.534 \pm 0.004$ \\
Period, $P$ & $4.02174 \pm 0.00003$ days \\
Inclination, $i$ & $72 \pm 1.5$ & \\
Eccentricity, $e$ & 0 (Fixed) & \\
Mass ratio $q$ & 0.75 (Fixed) \\
Temperature ratio & $0.85 \pm 0.02$ & \\
Semi-major axis $\left(R_{\odot}\right)$ & $50 \pm 1$ & \\
Systemic velocity & $-43 \pm 4$ km s & \\
\hline Parameter & Primary & Secondary \\
\hline Mass $\left(M_{\odot}\right)$ & $60 \pm 5$ & $45 \pm 4$ \\
Radius $\left(R_{\odot}\right)$ & $22 \pm 1$ & $19 \pm 1$ \\
log $g$ (cgs) & $3.53 \pm 0.01$ & $3.52 \pm 0.01$ \\
RV Semi-amplitude $\left(\mathrm{km} \mathrm{s}^{-1}\right)$ & $257 \pm 7$ & $343 \pm 9$ \\
mean $T_{\text {eff }}(\mathrm{K})$ & 35000 (Fixed) & $29900 \pm 700$ \\
Surface potential $(\Omega)$ & $3.14 \pm 0.02$ & 3.14 (Fixed) \\
$M_{\text {Bol }}$ & $-9.8 \pm 1$ & $-8.8 \pm 1$ \\
\hline
\end{tabular}

Notes. The errors shown should be considered internal errors of the fit. Any possible systematic errors are not included.

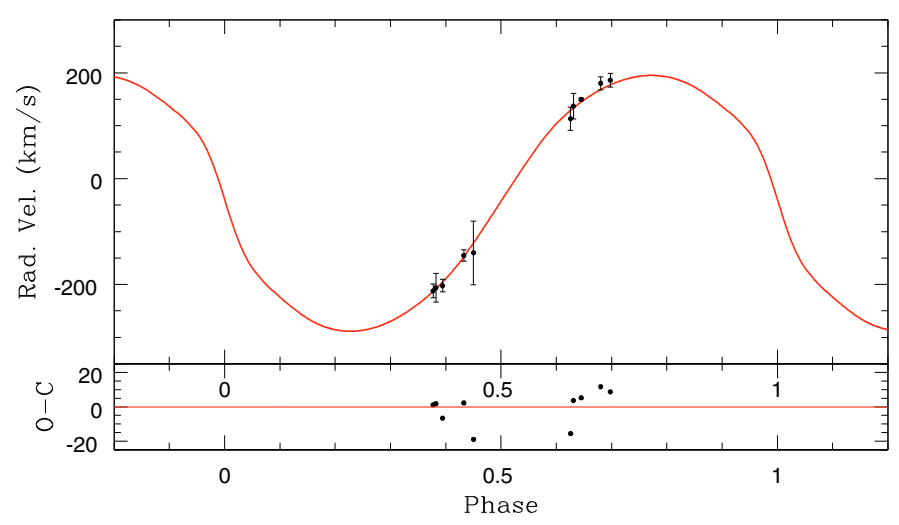

Fig. 9. The RV curve for the primary component obtained from the WHT data, with the best fit overlaid (solid line). The panel below shows the residuals between the observed data and the model.

With the absolute $V$ magnitudes, and the $V$ band reddening for B17, along with the $V$ band values for the two minima in the light curve, the distance modulus was calculated to be 10.9-11.3 which corresponds to a distance of about $1.5-1.8 \mathrm{kpc}$. While we regard these values as provisional due to the difficulties in modelling described above, these are consistent with the commonly adopted distance estimate of $1.7 \mathrm{kpc}$ (e.g. Hanson 2003; Torres-Dodgen et al. 1991; and Kiminki et al. 2007). Therefore they do not agree with the distance estimate of 900-950 pc obtained by Linder et al. (2009) from the light curve modelling of Cyg OB2 \#5.

A distance of $900 \mathrm{pc}$ would imply radii that are $\sim$ half those currently derived from the modelling. The derived radii scale linearly with the semi-major axis and the semi-major axis in turn is strongly dependant on the assumed mass ratio. For this system, a semi-major axis a factor of 2 smaller would imply a mass ratio of 6 . We consider this to be extremely unlikely, but not impossible.

\section{Discussion}

Given the difficulties in determining a unique model fit to the data (Sect. 5), we regard the parameters presented in Table 2 to be provisional. As a check on consistency we have calculated a preliminary bolometric magnitude for the components, following a similar procedure the that employed by Negueruela et al. (2008). Assuming that both components are of similar colour ${ }^{6}$, we adopted the 2 MASS value for $\left(J-K_{\mathrm{S}}\right)$ used in Negueruela et al. (2008), the effective temperature $T_{\text {eff }}$ and bolometric correction (BC) calibrations of Martins et al. (2005), and the intrinsic $(J-V)_{0}$ and $(K-V)_{0}$ colour calibrations of Wegner (1994). Using the 2MASS observed $\left(J-K_{\mathrm{S}}\right)$, we derived $E\left(J-K_{S}\right)$. The reddening of the system was calculated using the relation $A_{K_{\mathrm{S}}}=0.67 E\left(J-K_{\mathrm{S}}\right)$, due to the reddening in the association being close to standard (Hanson 2003). The reddening in the $V$ band was calculated using the relation $0.112 A_{V} \simeq A_{K}$ (Rieke \& Lebofsky 1985). The absolute $V$ band magnitude for the primary was then calculated, using the $V$ band value of the secondary minimum and adopting the distance modulus of 11.3, obtained by averaging spectroscopic distances (Kiminki et al. 2007). A semi-observational $M_{\text {bol }}$ was then calculated to be $-9.8 \pm 0.2$ and $-9.2 \pm 0.2$ for the primary and secondary, by adding $(V-K)_{0}$ and the BC to the $V_{0}$, assuming an uncertainty of one spectral type. With this value, the luminosities were calculated to be $\log \left(L_{1} / L_{\odot}\right)=5.8 \pm 0.1$ and $\log \left(L_{2} / L_{\odot}\right)=5.6 \pm 0.1$, noting that the main source of error is likely to be the uncertainty in the spectral type and hence temperature and $\mathrm{BC}$. We regard these estimates as upper limits since it assumes that both components are completely eclipsed during the minima.

The positions of the two components of B17 in the HR diagram (Fig. 10) are consistent with other known members of Cyg OB2 suggesting that it too is a bona fide member, with an age of 2.5 Myr (Negueruela et al. 2008).

With a likely spectral type of O9 Iaf, the secondary appears slightly more evolved than the primary and hence was likely the initially more massive star, with both evolving from very early $\mathrm{O}$ main sequence stars. We note that a system composed of O7 Iaf \& O9 Iaf stars is also compatible with the other massive evolved binaries in Cyg OB2. Indeed, the 6.6 day Ofpe/WNL+O6.5-7 binary Cyg OB2 \#5 (Linder et al. 2009) appears to be remarkably similar to B17, although with the Ofpe/WNL star being slightly more evolved than the O9 Iaf secondary in B17. The slightly longer period of Cyg OB2 \#5 can be explained by one or more from (i) initial birth parameters, (ii) mass transfer from one star to the other and (iii) mass lost by both stars via stellar winds.

Martins et al. (2007, 2008) used observations of the Galactic Centre and Arches clusters to examine the evolutionary pathways of stars at galactic metallicity with masses in excess of $30 M_{\odot}$, suggesting the following evolutionary pathways (also see Crowther et al. 1995):

- $\sim 30-60 M_{\odot}: \mathrm{O} \rightarrow$ Ofpe/WN9 $\rightleftharpoons \mathrm{LBV} \rightarrow \mathrm{WN} 8 \rightarrow \mathrm{WN} / \mathrm{C}$;

- $\sim 60-120 M_{\odot}: \mathrm{O} \rightarrow \mathrm{Of} \rightarrow \mathrm{WNL}+$ abs $\rightarrow \mathrm{WN7}$.

With an age of $2.5 \mathrm{Myr}$ and spectral classifications of $\mathrm{O} 7$ and O9, B17 appears to lie at the dividing point between the 2 evolutionary pathways. However, given that it appears significantly less massive than the WN6ha + WN6ha binary WR20a ( $m_{1}=m_{2}=83 M_{\odot}$, Rauw et al. 2004; Bonanos et al. 2004) we suspect that it will instead evolve to resemble Cyg OB2 \#5 and hence to a configuration similar to GCIRS 16SW, composed

\footnotetext{
${ }^{6}$ The colour differences between an $\mathrm{O} 7$ and an $\mathrm{O} 9$ supergiant are $(J-$ $V)_{0}=0.09$ and $(K-V)_{0}=0.11$ (Wegner 1994).
} 
Table 3. Evolved massive binaries in Cyg OB2 from Kiminki et al. (2009).

\begin{tabular}{llll}
\hline \hline Star & Sp Types & Period (days) & References \\
\hline MT05 & O9 III \& mid B & $25.1399(0.0008)$ & Kiminki et al. (2009) \\
MT720 & early B E early B & $<5$ & Kiminki et al. (2009) \\
Schulte 3 & O6 IV \&O9 III & $4.7464(0.0002)$ & Kiminki et al. (2009), Kinemuchi et al. (in prep.) \\
Schulte 5 (\#5) & O7 I \& Ofpe/WN9 & 6.6 & Wilson (1948), Wilson \& Abt (1951), Miczaika (1953) \\
& & & Walborn (1973), Contreras et al. (1997), Rauw et al. (1999) \\
Schulte 8a (\#8a) & O6 If \& O5.5 III(f) & 21.9 & Romano (1969), De Becker et al. (2004) \\
Schulte 9 (\#9) & O5.5 If \& O6-7 & 2.35 yrs & Nazé et al. (2008) \\
Schulte 73 & O8 II \& O8? & $17.4(0.2)$ & Kiminki et al. (2009) \\
B17 & O7 Ia \& O9 I & $4.0217(0.0004)$ & This paper \\
\hline
\end{tabular}

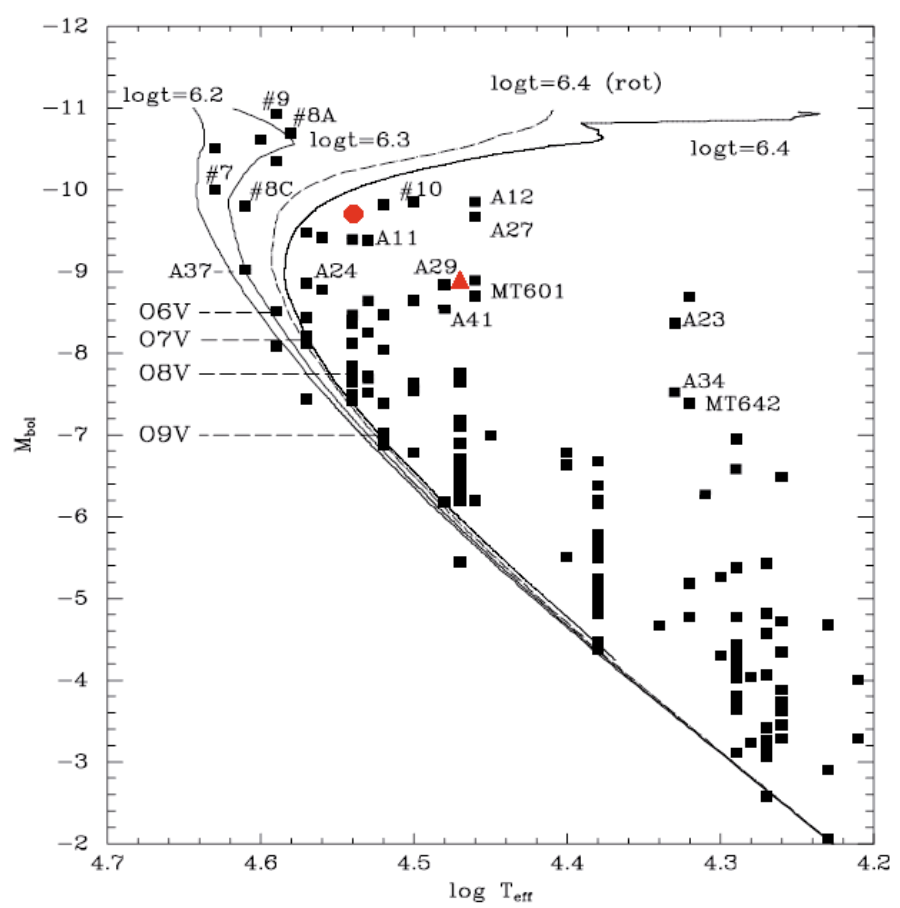

Fig. 10. Updated semi-observational HR diagram from Negueruela et al. (2008), based on published spectral classes and 2MASS $J H K_{\mathrm{S}}$ photometry and a DM of 11.3. The continuous lines are non-rotating isochrones for $\log t=6.2,6.3$ and 6.4 from Schaller et al. (1992) and the dashed line is the $\log t=6.4$ isochrone in the high-rotation models from Meynet \& Maeder (2003). The circle and the triangle show the positions of the primary and secondary components of B17.

of two cooler extreme B supergiants/LBV candidates $\left(P_{\text {orb }}=\right.$ 19.45 days; Martins et al. 2006), with stellar mass loss resulting in an eventual lengthening of the orbital period of B17. Indeed, assuming the system avoids merger during the LBV phase and, remaining bound, receives a favourable $\mathrm{SNe}$ kick to reduce the orbital separation it might briefly form a high mass X-ray binary with a WR mass donor prior to the second SN.

Irrespective of its ultimate fate, B17 is amongst the brightest/most massive systems in Cyg OB2 and adds to the increasing number of massive binaries identified within it (Kiminki et al. 2009, see Table 3). Similar trends for both a high binary fraction and the multiplicity of the brightest/most evolved cluster members have been observed for Pismis 24 (Maíz Apellániz 2008), NGC 3603 (Schnurr et al. 2008), Westerlund 1 (Clark et al. 2008; Ritchie et al. 2009) and potentially the Arches (Clark et al. 2009). If these trends continue it will have significant implications for the formation channels and relative production rates of both low and high mass X-ray binaries and systems comprising of two relativistic objects (Kobulnicky \& Fryer 2007).

\section{Summary}

Using photometric and spectroscopic data, we have demonstrated that B17 is an eclipsing, double lined spectroscopic binary comprising two supergiants with preliminary classifications of O7Iaf and O9Iaf. The spectra are highly variable, and with a subset revealing features from both stars, raise the possibility of achieving a dynamical mass determination for both components. Utilising both the photometric light curve and our limited $\mathrm{RV}$ dataset we attempted to determine an initial orbital solution for the binary.

Despite the morphology of the light curve indicating a semicontact configuration we were unable to achieve convergence for such a hypothesis and hence were forced to adopted an overcontact configuration. In the absence of a full RV curve for both system components we were forced to fix the binary mass ratio, and had to include the presence of a star spot to address the observed asymmetries in the light curve (which are likely due to the effects of binary mass transfer). However, we were still unable to fully fit both secondary eclipse and the light curve between orbital phase $\sim 0.6-0.9$ with such a model; as such we regard the modelling results presented in this work as provisional. We anticipate that a full RV curve for both components will be necessary to obtain more precise parameters for the system; additional data to accomplish this goal are currently being obtained and refined analysis will be presented in a future paper.

Nevertheless, the provisional distance calculation of $1.5-1.8 \mathrm{kpc}$ obtained from the light curve analysis agrees with previously published values for the distance to $\mathrm{Cyg} \mathrm{OB} 2$, being inconsistent with the distance of 900-950 pc determined by Linder et al. (2009) with the Cyg OB2 \#5 light curve analysis.

When placed in the HR diagram, B17 appears to be consistent with the age and stellar population of the Cyg OB2 association. Assuming the system avoids merger, it is likely to evolve through an extreme B supergiant/LBV phase into a long period WR+WR binary configuration as mass loss via stellar winds increases the orbital separation. In combination with the recent work of Kiminki et al. (2009) and Kobulnicky \& Fryer (2007) the results of our analysis provides additional evidence that Cyg OB2 has a very high fraction of massive binary stars. Such an observational constraint needs to be considered when determining the initial mass function of the association as it may both influence the slope of the relationship and also lead to a population of artificially massive stars, resulting in the inflation of a putative high mass cut-off to the IMF. 
Acknowledgements. We thank Pedro Pastor and Manuel Méndez for having obtained and reduced the photometric data. Amparo Marco for help with the 2004 observing run and Miriam García for assistance with the 2006 INT run. We thank Dan Kiminki, Fraser Lewis and Chris Evans for useful discussions and reading of the manuscript. We also thank the referee for his guidance in completing the paper for publication. The Faulkes Telescope Project is an educational and research arm of the Las Cumbres Observatory Global Telescope Network (LCOGT). V.S. acknowledges support from the Dill Faulkes Educational Trust. This research is partially supported by the Spanish Ministerio de Ciencia e Innovación undergrants AYA2008-06166-C03-03 and Consolider-GTC CSD2006-70. The G.D. Cassini telescope is operated at the Loiano Observatory by the Osservatorio Astronomico di Bologna. The WHT is operated on the island of La Palma by the Isaac Newton Group in the Spanish Observatorio del Roque de Los Muchachos of the Instituto de Astrofísica de Canarias. The 2006 observations were taken as part of the service programme (programme SW2005A20).

\section{References}

Bertin, E., \& Arnouts, S. 1996, A\&A, 117, 393

Bonanos, A. Z. 2009, ApJ, 691, 407

Bonanos, A. Z., Stanek, K. Z., Udalski, A., et al. 2004, ApJ, 611, L33

Clark, J. S., Negueruela, I., Crowther, P. A., \& Goodwin, S. P. 2005, A\&A, 434, 949

Clark, J. S., Muno, M. P., Negueruela, I., et al. 2008, A\&A, 477, 147

Clark, J. S., Crowther, P. A., \& Mikles, V. J. 2009, A\&A, 507, 1567

Comerón, F., Pasquali, A., Rodighiero, G., et al. 2002, A\&A, 389, 874

Contreras, M. E., Rodriguez, L. F., Tapia, M., et al. 1997, ApJ, 488, 153

Crowther, P. A., Smith, L. J., Hillier, D. J., \& Schmutz, W. 1995, A\&A, 293, 427

De Becker, M., Rauw, G., \& Manfroid, J. 2004, A\&A, 424, L39

Dhillon, V. S., Privett, G. J., \& Duffey, K. P. 2001, Starlink User Note 167.6, R.A.L

Draper, P. W., Taylor, M., \& Allan, A. 2000, Starlink User Note 139.12, R.A.L

Figer, D. F. 2005, Nature, 434, 192

Figer, D. F., McLean, I. S., \& Morris, M. 1999, ApJ, 514, 202

Gies D. R. 2002, IAU Symp., 212, 91

Hanson, M. M. 2003, ApJ, 597, 957

Hilditch, R. W., Howarth, I. D., \& Harries, T. J. 2005, MNRAS, 357, 304

Howarth, I., Murray, J., Mills, D., \& Berry, D. S. 1998, Starlink User Note 50.21, R.A.L.

Kiminki, D. C., Kobulnicky H. A., \& Kinemuchi K., et al. 2007, ApJ, 664, 1102 Kiminki, D. C., McSwain V., \& Kobulnicky H. A. 2008, ApJ, 679, 1478
Kiminki, D. C., Kobulnicky, H. A., Gilbert, I., Bird, S., \& Chunev, G. 2009, AJ, 137,4680

Knödlseder, J. 2000, A\&A, 360, 539

Kobulnicky, H. A., \& Fryer, C. L. 2007, ApJ, 670, 747

Linder, N., Rauw, G., Manfroid, J., et al. 2009, A\&A, 495, 231

Maíz Apellániz, J. 2008, ApJ, 677, 1278

Martins, F., Schaerer, D., \& Hillier, D. J. 2005, A\&A, 436, 1049

Martins, F., Trippe, S., Paumard, T., et al. 2006, ApJ, 649, L103

Martins, F., Genzel, R., Hillier, D. J., et al. 2007, A\&A, 468, 233

Martins, F., Hillier, D. J., Paumard, T., et al. 2008, A\&A, 478, 219

Massey, P., \& Thompson, A. B. 1991, AJ, 101, 1408

Meynet, G., \& Maeder, A. 2003, A\&A, 404, 975

Miczaika, G. R. 1953, PASP, 65, 141

Nazé, Y., De Becker, M., Rauw, G., \& Barbieri, C. 2008, A\&A, 483, 543

Negueruela, I., Marco, A., Herrero A., \& Clark, J. S. 2008, A\&A, 487, 575

Otero, S. 2008, Open European Journal on Variable Stars, 91

Prinja, R. K., Barlow, M. J., \& Howarth, I. D. 1990, ApJ, 361, 607

Rauw, G., Vreux, J.-M., \& Bohannan, B. 1999, ApJ, 517, 416

Rauw, G., De Becker, M., Nazé, Y., et al. 2004, A\&A, 420, L9

Ribas, I. 2006, ASPC, 349, 55

Rieke, G. H., \& Lebofsky, M. J. 1985, ApJ, 288, 618

Ritchie, B. W., Clark, J. S., Negueruela, I., \& Crowthter, P. A. 2009, A\&A, 507, 1585

Romano, G. 1969, MmSAI, 40, 375

Sana, H., Gosset, E., Nazé, Y., Rauw, G., \& Linder, N. 2008, MNRAS, 386, 447S

Schaller, G., Schaerer, D., Meynet, G., \& Maeder, A. 1992, A\&AS, 96, 269

Schnurr, O., Casoli, J., Chené, A.-N., Moffat, A. F. J., \& St-Louis, N. 2008, MNRAS, 389, L38

Shortridge, K., Meyerdicks, H., Currie, M., et al. 1997, Starlink User Note 86.15, R.A.L

Torres-Dodgen, A. V., Carroll, M., \& Tapia, M. 1991, MNRAS, 249, 1

Walborn, N. R. 1973, ApJ, 180, L35

Walborn, N. R., \& Fitzpatrick, E. L. 1990, PASP, 102, 379

Walborn, N. R., \& Howarth I. D. 2000, PASP, 112, 1446

Walborn, N. 2001, in Eta Carinae and Other Mysterious Stars: The Hidden Opportunities of Emission Spectroscopy, ed. T. R. Gull, S. Johannson, \& K. Davidson, ASP Conf. Ser., 242, 217

Wegner, W. 1994, MNRAS, 270, 229

Wilson, O. C. 1948, PASP, 60385

Wilson, O. C., \& Abt, A. 1951, ApJ, 144, 477

Wilson, R. E., \& Devinney, E. J. 1971, ApJ, 166, 605

Wózniak, P. R., Vestrand W. T., Akerlof C. W., et al. 2004, ApJ, 127, 2434 